\title{
NATURALEZA Y LÓGICA DE LAS PROPOSICIONES NORMATIVAS. CONTRIBUCIÓN EN HOMENAJE A G. H. VON WRIGHT
}

\author{
Jorge L. Rodríguez \\ Universidad Nacional de Mar del Plata
}

\section{Introducción*}

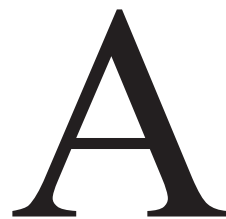

1 examinar el discurso normativo y su lógica subyacente, una de las cuestiones de mayor importancia es la necesidad de apreciar con claridad las consecuencias que se siguen de la distinción, destacada entre otros por von Wright, entre normas y proposiciones normativas. Las normas son expresadas por formulaciones normativas; constituyen el significado de oraciones usadas para prescribir, esto es, para ordenar, prohibir o permitir ciertas conductas. Las proposiciones normativas, en cambio, constituirían el significado de oraciones que califican ciertas acciones como obligatorias, prohibidas o permitidas de conformidad con ciertas normas. ${ }^{1}$ Mientras que las proposiciones normativas son susceptibles de verdad o falsedad, no parece tener mayor sentido predicar tal cosa de las propias normas. La dificultad para distinguir estas dos nociones deviene de que la misma oración (por ejemplo, "Está prohibido fumar") puede a veces ser utilizada para formular una norma y otras para referirse a la existencia de una norma, esto es, puede a veces expresar una norma y otras una proposición normativa. Es verdaderamente lamentable que, pese a que con denominaciones diversas la diferencia que esta distinción recoge ha sido reconocida por muchos autores, ella no ha recibido todavía hoy la atención que a mi juicio merece en el terreno de la lógica del discurso normativo. ${ }^{2}$

\footnotetext{
* Quiero expresar mi profundo agradecimiento a Pablo Navarro, a quien debo la sugerencia de desarrollar las ideas que he tratado de volcar en las dos últimas secciones del trabajo.

${ }^{1}$ Cf. G. H. von Wright, Norma y acción. Una investigación lógica, Madrid, Tecnos, 1970, título original: Norm and Action. A Logical Inquiry, London, Routledge \& Kegan Paul, 1963, página 109.

${ }^{2}$ Cf. G. H von Wright, "Deontic Logic - As I See It", en P. McNamara y H. Prakken, Norms, Logics and Information Systems, Amsterdam-Berlin-Oxford-Tokyo-Washington DC, IOS Press, 1999, página 20.
} 
En el presente trabajo intentaré examinar tres cuestiones vinculadas con las características distintivas de las proposiciones normativas y su lógica. En primer lugar, la posibilidad de concebir, junto a proposiciones normativas relativas a un cierto conjunto de normas, proposiciones normativas de carácter absoluto, esto es, proposiciones que califican normativamente ciertas acciones como obligatorias, prohibidas o permitidas no relativizadas con relación a un determinado conjunto de normas. En segundo lugar, la posibilidad de diferenciar la categoría de los "enunciados normativos", entendidos como enunciados acerca de que algo debe o puede ser hecho, de la categoría de las proposiciones normativas, esto es, proposiciones relativas a la existencia de una norma. Por último, trataré de reseñar las particularidades de dos visiones alternativas de reconstrucción de la lógica de proposiciones normativas, así como de los presupuestos subyacentes a cada una de ellas.

\section{El carácter relativo de las proposiciones normativas}

A primera vista, los dos rasgos más salientes que justifican diferenciar a las proposiciones normativas de las normas son, en primer lugar, que aunque es altamente dudoso que pueda predicarse verdad o falsedad de las normas, tales calificaciones sí cobran pleno sentido respecto de las proposiciones normativas. Y, en segundo lugar, que las proposiciones normativas son relativas. Una misma acción $\mathrm{p}$ puede estar prohibida, ser permitida $\mathrm{u}$ obligatoria, en un cierto sistema normativo y no en otro. Por tal motivo, la expresión "p está prohibido", entendida como una proposición normativa, es incompleta, ya que mientras no se especifique de qué sistema normativo se trata, carecerá de valor de verdad. ${ }^{3}$

Este segundo rasgo de las proposiciones normativas ha sido, con todo, controvertido recientemente por Jan-R. Sieckmann. ${ }^{4}$ Sieckmann sostiene que una proposición normativa, entendida como la afirmación de que una norma es válida de conformidad con cierto sistema de normas, es una afirmación relativa a su pertenencia al sistema en cuestión, pero no un enunciado que de manera directa predique que una norma es "válida", entendiendo aquí esta última expresión en sentido prescriptivo, esto es, como referida a que deba hacerse lo que ella dispone. A su criterio, un enunciado normativo tal como "No se debe fumar" contendría una proposición normativa relativa a que no se debe fumar, lo cual se revelaría en la posibilidad de que el hablante

${ }^{3}$ Cf. E. Bulygin "Lógica deóntica”, en C. Alchourrón et al. (eds.), Enciclopedia Iberoamericana de Filosofía, Volumen 7, Lógica, Madrid, Trotta, 1995, páginas 129-142.

${ }^{4}$ Cf. J. R. Sieckmann, "Remarks on Jorge Rodríguez's 'Sistemas Jurídicos y Derrotabilidad del Derecho'”, 2000, inédito y J. R. Sieckmann, “The Fragmentation of Deontic Logic”, ponencia presentada en el XIX Congreso Mundial de Filosofía del Derecho y Filosofía Social, Lundt, 2003. 
ponga de manifiesto su carácter proposicional en oraciones como "Es el caso que no se debe fumar" o "Es verdad que no se debe fumar". Pero Sieckmann sostiene además que si se asume que no es posible predicar verdad o falsedad de las normas, las proposiciones normativas sólo podrían interpretarse como aquello que expresan oraciones que se refieren a la pertenencia de una norma a un cierto sistema, y esto último le parece inaceptable por cuanto, si bien muchas veces se formulan enunciados normativos relativos a un cierto sistema, la forma más elemental de una proposición normativa sería a su juicio una afirmación directa o absoluta de lo que es obligatorio, prohibido o permitido.

Para Sieckmann, la necesidad de una lógica de proposiciones normativas en forma pura o absoluta se revelaría con claridad cuando se intenta responder a la pregunta acerca de qué se debe hacer en cierta situación, en el sentido de qué norma debe guiar nuestras acciones. La respuesta a esta pregunta requeriría de una proposición normativa absoluta, ya que un enunciado que dijese que de conformidad con cierto sistema normativo se debe hacer $p$ sería manifiestamente insuficiente puesto que podría aceptarse su verdad y, no obstante, preguntarse si realmente debe seguirse esa norma. En cambio, si una proposición normativa absoluta es verdadera, no podría seriamente cuestionarse si ha de seguirse la norma a la que ella alude.

Desde mi punto de vista, la relatividad a un cierto sistema normativo es la característica más significativa de las proposiciones normativas, de manera que esta categoría de proposiciones normativas absolutas que Sieckmann introduce no puede sino ser el producto de una confusión porque resulta autocontradictoria. Intentaré justificar esta idea. En una primera interpretación, la idea de una proposición normativa absoluta podría entenderse como una proposición referida a una norma de validez absoluta. Bajo esta interpretación, el calificativo de "absolutas" que Sieckmann atribuye a las proposiciones normativas no sería sino un referencia al tipo de normas a cuyo respecto se formulan tales proposiciones. Ello, claro está, si es que puede asignarse sentido a la expresión "norma absoluta", cualquiera sea éste. Pero entonces las proposiciones normativas absolutas serían aquellas proposiciones normativas relativas al conjunto de las normas absolutas. No existiría ninguna diferencia significativa entre este tipo de entidades y las proposiciones normativas relativas a cierto sistema, pues la única peculiaridad se encontraría aquí en el dominio de las normas que se toman como punto de referencia. En particular, los problemas que pudiera suscitar la identificación de "normas absolutas" semejantes resultarían independientes de las relaciones entre las proposiciones que a ellas se refieren.

Las mismas distinciones que correspondería establecer en la lógica de proposiciones normativas ordinaria se reproducirían respecto de las "pro- 
posiciones normativas absolutas" así entendidas. Por ejemplo, aquella que media entre el sentido positivo y el sentido negativo de la permisión. Como veremos, puede decirse que una conducta está permitida en sentido positivo de acuerdo con las normas de cierto sistema normativo si, y sólo si, en él existe una norma que permite dicha conducta. En cambio, una conducta está permitida en sentido meramente negativo de acuerdo con las normas de cierto sistema normativo si, y sólo si, no existe en el sistema de referencia una norma que prohíba tal conducta. Como fuera demostrado por Carlos Alchourrón, la permisión positiva y la permisión negativa sólo pueden considerarse equivalentes respecto de sistemas normativos completos (esto es, que no contienen lagunas) y consistentes (es decir, que no contienen contradicciones). ${ }^{5}$ Pero, a excepción de ese supuesto, ambas nociones no pueden asimilarse. En razón de esta ambigüedad en la expresión "permitido" cuando aparece en proposiciones normativas, que no se verifica cuando tal expresión es usada para formular una norma (contexto en el cual "permitido" equivale a "no prohibido"), es que una lógica de normas no resulta equivalente a una lógica de proposiciones normativas. Todo esto, como se dijo, valdría igualmente para proposiciones normativas relativas a "normas absolutas", razón por la cual no podría fundarse sobre esta interpretación la pretensión de distinguir a las proposiciones normativas absolutas como entidades conceptualmente distintas de las proposiciones normativas -relativas- ordinarias.

Descartada esta posibilidad, una interpretación alternativa consistiría en considerar que el calificativo de "absolutas" no se refiere a las normas mencionadas en las proposiciones normativas absolutas, sino a las propias proposiciones normativas. Una proposición normativa relativa calificaría normativamente una acción de conformidad con un cierto sistema normativo. A diferencia de ella, una proposición normativa absoluta sería aquella que califica normativamente a una acción considerando todos los factores relevantes (all things considered). A primera vista, diría que determinar la calificación normativa de una acción considerando todos los factores relevantes sería tanto como hacerlo tomando en cuenta, no un cierto sistema normativo, sino una pluralidad de sistemas normativos. En tal sentido, me parece que la perspectiva intersistemática es conceptualmente dependiente de la intrasistemática. ${ }^{6}$ Pero examinemos más detenidamente esta alternativa.

${ }^{5}$ Cf. C. E. Alchourrón, "Logic of Norms and Logic of Normative Propositions", en Logique et Analyse 12, N 47, 1969, páginas 242-268.

${ }^{6}$ Para un punto de vista semejante en la discusión en torno a la derrotabilidad, véase M. Belzer y B. Loewer, "Deontic Logics of Defeasibility", en D. Nute (ed.), Defeasible Deontic Logic, Dordrecht-Boston-London, Kluwer Academic Publishers, 1997, página 45. 
Una forma de entender esta idea sería decir que las proposiciones normativas absolutas son proposiciones normativas relativas a la totalidad de los sistemas normativos relevantes. Pero para Sieckmann las proposiciones normativas absolutas deben suministrar una respuesta a la pregunta acerca de qué se debe hacer en cierta situación. Supóngase que quiero saber si debo o no realizar la acción p en la situación $C$; que dos normas resultan relevantes en tal situación -una norma jurídica y una norma moral, por ejemplo-, y que cada una de ellas brinda una respuesta diferente. Es evidente que una proposición normativa que se limitase a informar acerca de una disyunción de normas potencialmente incompatibles no respondería a la pregunta acerca de lo que se debe hacer en $C$. Lo que se requeriría es un criterio de selección entre las diferentes normas en juego. En otras palabras, la idea de una proposición normativa absoluta, tal como la concibe Sieckmann, no sólo exige tomar en cuenta todos los sistemas relevantes, sino además las relaciones de prelación o jerarquía entre las normas de tales sistemas. Las relaciones de preferencia entre normas de diferentes sistemas normativos pueden asumir formas diversas. Puede que un sistema normativo $S_{1}$ sea jerárquicamente superior a otro $\mathrm{S}_{2}$ como un todo, esto es, que dada una norma cualquiera de $S_{1}$ y una norma cualquiera de $S_{2}$, siempre se prefiera la primera sobre la segunda. Puede ocurrir, en cambio, que algunas normas de $\mathrm{S}_{1}$ sean preferidas a algunas normas de $\mathrm{S}_{2}$, pero que asimismo algunas normas de $\mathrm{S}_{2}$ sean preferidas a algunas normas de $\mathrm{S}_{1}$. $\mathrm{Y}$ también puede ocurrir que ciertas normas de $\mathrm{S}_{1}$ sean en ciertos casos preferidas a ciertas normas de $\mathrm{S}_{2}$, mientras que en otros se prefiera a las de $S_{2}$ sobre las de $S_{1}$.

De todos modos, sumar a la totalidad de los sistemas relevantes las relaciones de preferencia entre sus diversas normas todavía sería insuficiente. Una proposición normativa absoluta sólo estaría en condiciones de ofrecer una respuesta definitiva a la pregunta acerca de lo que se debe hacer en la situación $\mathrm{C}$ si, además de tomar en cuenta todos los sistemas relevantes y las relaciones de preferencia entre sus normas, se cumpliera que tales criterios permiten seleccionar una y sólo una norma como aquella que resulta aplicable en C. La simple existencia de criterios de preferencia no garantiza que su empleo permita privilegiar a una única norma sobre las restantes. Considerar que frente a cualquier situación $\mathrm{C}$ es posible formular una proposición normativa absoluta requiere presuponer que existe al menos una norma que regula la cuestión y, en caso de que haya más de una, que es posible resolver cualquier posible conflicto entre las que se presentan como prima facie relevantes.

La pregunta que cabe formular es si, así interpretadas, estas "proposiciones normativas absolutas" tendrían el carácter de genuinas proposiciones normativas o más bien el de normas. Si lo que interesa es resolver el 
problema práctico de qué se debe hacer en la situación $\mathrm{C}$, puede resultar valioso que se informe sobre la existencia de una norma. Ello por cuanto la ignorancia acerca de su existencia puede llevar a escoger una solución equivocada. En este sentido, puede decirse que una proposición normativa, esto es, la información sobre la existencia de una norma, puede -al menos en ciertos casos- tener incidencia en la resolución de un problema práctico. Pero suministrar información no resulta suficiente para resolver un problema práctico, dado que frente a la proposición que afirma la existencia de una norma siempre cabe la pregunta ¿se debe hacer lo que esta norma prescribe? Lo que se requiere para resolver un problema práctico es usar una norma. $\mathrm{Y}$ si en una cierta situación $\mathrm{C}$ concurren varias normas como relevantes, se debe elegir a una de ellas como guía para la acción. En otras palabras, bajo la interpretación aquí considerada, una proposición normativa absoluta sería aquella que, efectuando un balance entre las distintas normas que se presentan como relevantes en la situación $\mathrm{C}$, privilegia a una de ellas como aquella que debe orientar la conducta. Ahora bien, si ese "debe" es descriptivo, esto es, si se limita a indicar la existencia de un criterio para escoger entre las normas en juego, todavía se hallaría abierta la posibilidad de cuestionar por qué ha de emplearse ese criterio de elección y no otro entre las diversas normas en juego. Si en cambio ese "debe" se interpreta prescriptivamente, el enunciado "Entre las diversas normas en juego frente a la situación $\mathrm{C}$, debe usted elegir la norma $\mathrm{N}$ como guía de su conducta" no expresaría una proposición normativa sino lisa y llanamente una norma. De manera que bajo esta interpretación, la idea de una proposición normativa absoluta no sería sino el producto de confundir a las proposiciones referidas a normas con las propias normas.

Aunque me parece destacable que Sieckmann tome en cuenta la distinción entre normas y proposiciones normativas, hablar de proposiciones normativas absolutas resulta inadecuado porque, o bien introduce un problema que debería mantenerse al margen de ella -el del fundamento del deber de cumplir las normas a las que las proposiciones normativas hacen referencia-, o bien conduce en última instancia a confundir lo que pretende distinguirse.

\section{Proposiciones normativas y enunciados normativos}

En un trabajo de 1965, von Wright se pregunta por los fundamentos de los enunciados normativos. ${ }^{7}$ Allí define "enunciado normativo" como un

${ }^{7}$ Cf. G. H. von Wright, "The Foundation of Norms and Normative Statements", publicado originariamente en K. Ajdukiewicz (ed.), The Foundations of Statements and Decisions, Warsawa, Polish Scientific Publishers, 1965, páginas 351-367 y posteriormente incluido en G. 
enunciado acerca de que algo debe o puede ser hecho. El fundamento de un enunciado normativo sería "el contenido de una respuesta verdadera a la pregunta acerca de por qué aquello a lo que se refiere el enunciado debe o puede ser hecho", por lo que también podría ser calificado como su fundamento de verdad. A veces quien formula el enunciado suministra él mismo un fundamento ("Se debe hacer p porque..."), pero si no se suministra un fundamento, puede inquirirse por él.

A juicio de von Wright, una posible respuesta a la pregunta acerca de por qué se debe hacer $\mathrm{p}$ es que existe una norma que establece que debe hacerse p, y ello constituiría el fundamento de verdad del enunciado normativo. Para von Wright, "norma" alude aquí a una prescripción o regulación, que un cierto agente (autoridad) da a otro (sujeto normativo), lo cual requiere el uso del lenguaje. Señala que las normas se diferencian de los enunciados normativos en el hecho de que respecto de las primeras no cabe predicar verdad o falsedad, mientras que decir que existe una norma es una afirmación susceptible de verdad o falsedad. Otras veces, el fundamento de verdad de un enunciado normativo podría deducirse de la existencia de una norma. En general, las normas se refieren a categorías o clases de actos, mientras que los enunciados normativos se refieren a actos individuales.

De estas consideraciones de von Wright puede concluirse que el "fundamento de verdad" de los enunciados normativos dependería siempre de la existencia de una norma, ya sea de manera directa o indirecta. Esto último ocurriría toda vez que la verdad del enunciado normativo dependiese, no directamente de lo que una norma diga, sino de una consecuencia lógica de ella. Así, podría afirmarse que los enunciados normativos expresan proposiciones normativas, esto es, proposiciones que predican la existencia de una norma, sea que ella haya sido directamente promulgada por cierta autoridad o que pueda derivarse de las promulgadas por cierta autoridad.

Aunque en el planteo que efectúa von Wright parece distinguirse entre enunciados normativos y proposiciones normativas, si se toma en cuenta lo dicho hasta aquí, un enunciado normativo no parecería más que una manera indirecta de referirse a la existencia de una norma. Bulygin ha elaborado con cierto detalle este punto de vista y, en un trabajo reciente, sostiene que enunciados como (1) "Usted no debe estacionar aquí" y (2) "Hay una norma que prohíbe estacionar aquí" tienen el mismo significado, siendo (2) una forma expandida de (1). Un enunciado como (2), que expresaría una proposición normativa en su forma expandida, tendría la ventaja de que ya no sería ambiguo. Ello en razón de que no sería posible usarlo para establecer una

H von Wright, Practical Reason - Philosophical Papers Volume I, Ithaca, New York, Cornell University Press, 1983, páginas 67-82. 
prohibición por tratarse de un enunciado característicamente descriptivo, mientras que el enunciado (1) sería, en cambio, ambiguo porque podría ser usado para formular una norma o expresar una proposición normativa. ${ }^{8}$

El fundamento de esta conclusión es que no existiría diferencia entre informar, por ejemplo, acerca de las regulaciones existentes respecto de la prohibición de estacionar, y afirmar que hay una norma que prohíbe estacionar. Si la información es verdadera, entonces existiría una norma que prohíbe estacionar. Si en cambio no existiese esa norma, la afirmación sería falsa. En su reconstrucción, Bulygin sostiene que una proposición normativa afirma que existe una norma en un cierto sistema normativo que regula una determinada acción. Por consiguiente, considera que un enunciado como (2) -"Hay una norma que prohíbe estacionar aquí"- tampoco exhibiría todo su contenido conceptual y debería ser expandido en un enunciado como:

(3) "En el sistema normativo $\alpha$ hay una norma que prohíbe estacionar aquí".

Desde este punto de vista, una proposición normativa establecería una relación entre una norma y un sistema normativo y, en tanto ambos términos de la relación no estuviesen determinados, no existiría proposición normativa.

Pablo Navarro ha propuesto denominar a los enunciados que predican la pertenencia de una norma a un sistema "proposiciones sistemáticas", distinguiéndolos de las proposiciones normativas, que afirmarían la existencia de una norma. ${ }^{9}$ Sobre tales bases, sostiene que la propuesta de Bulygin equivaldría a identificar los enunciados normativos con proposiciones normativas y, a su vez, estas últimas con proposiciones sistemáticas. Para Bulygin, los enunciados normativos sólo serían una forma elíptica de afirmar una proposición sistemática, un modo transpuesto de discurso (transposed mode of speech), pues para decir que hay una norma que prohíbe una acción p en un cierto sistema normativo, se diría que p está prohibida.

A juicio de Navarro, el trabajo de Bulygin sólo probaría que los enunciados normativos tienen las mismas condiciones de verdad que las proposiciones normativas, pero ello no garantizaría que tuviesen el mismo significado, y menos aún que tuviesen el mismo significado que las proposiciones sistemáticas. Por mi parte, considero que si bien la primera observación de Navarro puede admitirse, la segunda es fruto de un malentendido. En efecto, en la sugerencia de Bulygin no hay un paso de enunciados normativos a

${ }^{8}$ Cf. E. Bulygin, "True or False Statements in Normative Discourse", en R. Eigidi (ed.), Search of a New Humanism: The Philosophy of G.H. von Wright, Synthese, v. 282, 1999.

${ }^{9}$ Cf. P. E. Navarro, "Legal Reasoning and Systematization of Law", presentado como ponencia al XX Congreso Mundial de Filosofia del Derecho y Filosofia Social, Amsterdam, 2001. 
proposiciones normativas y uno adicional a "proposiciones sistemáticas". Identificar un enunciado que se refiere a la existencia de una norma con uno que predica la pertenencia de una norma a un cierto sistema no es más que una forma de hacer referencia a que las calificaciones normativas de ciertas acciones no suelen hacerse tomando en cuenta una única norma sino una pluralidad de ellas. Pero aún cuando no se asumiera este último presupuesto, desde un punto de vista estrictamente formal parece más adecuado relativizar la noción de proposición normativa a un sistema normativo en lugar de hacerlo respecto de una norma, dado que esto último puede ser visto como un caso límite de lo primero, esto es, un sistema normativo conformado por una única norma. Por ello, considero que el paso de (2) a (3) es trivial. Por su parte, el paso de (1) a (2) me parece plenamente justificado siempre que (1) sea interpretado descriptivamente, ya que la aparente diferencia en el significado entre uno y otro no es sino el fruto de la ambigüedad descriptivo/prescriptivo que afecta a (1) pero no a (2).

\section{Lógica de normas y lógica de proposiciones normativas}

En el sistema clásico de lógica deóntica ideado por von Wright ${ }^{10}$ se toman como interdefinibles a los conceptos de prohibición, obligación y permisión:
1. $\mathrm{Op}=\operatorname{def} \mathrm{PH} \sim \mathrm{p}$
3. $\mathrm{PHp}=\operatorname{def} \mathrm{O} \sim \mathrm{p}$
2. $\mathrm{PHp}=\operatorname{def} \sim \mathrm{Pp}$
4. $\mathrm{Op}=\operatorname{def} \sim \mathrm{P} \sim \mathrm{p}$

En una presentación axiomática del sistema tendríamos los siguientes tres axiomas:

\section{Sistema LN:}

$$
\begin{array}{ll}
\left(\mathrm{A}_{0}\right) & \mid-\mathrm{Pp} \leftrightarrow \sim \mathrm{O} \sim \mathrm{p} \\
\left(\mathrm{A}_{1}\right) & -\mathrm{O}(\mathrm{p} \wedge \mathrm{q}) \leftrightarrow(\mathrm{Op} \wedge \mathrm{Oq}) \\
\left(\mathrm{A}_{2}\right) & -\mathrm{Op} \rightarrow \mathrm{Pp}
\end{array}
$$

Se acepta además la siguiente regla de inferencia:

$$
\text { De } \mid-(p \leftrightarrow q) \text {, se sigue } \mid-(O p \leftrightarrow O q)^{11}
$$

${ }^{10}$ Cf. G. H. von Wright, "Deontic Logic”, en Mind 60, 1951, páginas 1-15.

${ }^{11}$ Cf. C. Alchourrón, "Philosophical Foundations of Deontic Logic and the Logic of Defeasible Conditionals", en J. Meyer y R. Wieringa, Deontic Logic in Computer Science: Normative System Specification, Chichester-New York-Brisbane-Toronto-Singapore, Wiley \& Sons, 1993, páginas 43-84. 
El primer axioma indica la interdefinibilidad entre permisión y obligación. El segundo, la distributividad de la obligación respecto de la conjunción. El tercero, el principio de que obligatorio implica permitido -equivalente al llamado principio de permisión: "Pp $\vee \mathrm{P} \sim \mathrm{p}$ "- . La regla de inferencia del sistema establece que toda tautología de la lógica de enunciados es una fórmula válida del sistema cuando las variables de enunciado son reemplazadas por fórmulas deónticas.

El axioma $\mathrm{A}_{0}$ garantiza la validez de las siguientes dos leyes:

5. $\sim(\mathrm{O} \sim \mathrm{p} \wedge \mathrm{Pp}) \quad$ principio de consistencia normativa

6. $\mathrm{O} \sim \mathrm{p} \vee \mathrm{Pp} \quad$ principio de completitud normativa

Demostración:

7. $\mathrm{Pp} \rightarrow \sim \mathrm{O} \sim \mathrm{p} \quad$ implicación de izquierda a derecha de $\mathrm{A}_{0}$.

8. $\mathrm{O} \sim \mathrm{p} \rightarrow \sim \mathrm{Pp} \quad$ contraposición en 7. $((\mathrm{p} \rightarrow \mathrm{q}) \rightarrow(\sim \mathrm{q} \rightarrow \sim \mathrm{p}))$

9. $\sim(\mathrm{O} \sim \mathrm{p} \wedge \mathrm{Pp}) \quad$ definición del condicional en $8 .((\mathrm{p} \rightarrow \mathrm{q}) \leftrightarrow \sim(\mathrm{p} \wedge \sim \mathrm{q}))$.

10. $\sim \mathrm{O} \sim \mathrm{p} \rightarrow \mathrm{Pp} \quad$ implicación de derecha a izquierda de $\mathrm{A}_{0}$.

11. $\mathrm{O} \sim \mathrm{p} \vee \mathrm{Pp} \quad$ definición del condicional en $10 .((\mathrm{p} \rightarrow \mathrm{q}) \leftrightarrow(\sim \mathrm{p} \vee \mathrm{q}))$.

De manera que de acuerdo con este sistema, respecto de cualquier acción, ella no podría estar prohibida y permitida a la vez (principio de consistencia normativa) y, o bien está prohibida, o bien está permitida (principio de completitud normativa).

¿Reconstruye este sistema acabadamente las relaciones lógicas entre expresiones como "obligatorio", "prohibido" y "permitido"? Para responder a esta pregunta debe tenerse presente la ya apuntada sistemática ambigüedad de tales expresiones, esto es, que ellas pueden aparecer tanto en normas en sentido genuino como en proposiciones normativas.

Resulta muy importante diferenciar claramente estas dos interpretaciones de expresiones como "obligatorio", "prohibido" y "permitido", así como de las oraciones en las que ellas aparecen. Una lógica de estas expresiones interpretadas prescriptivamente (una genuina lógica de normas) pretenderá reconstruir la racionalidad en la actividad de dictar normas, la "racionalidad del legislador". En cambio, una lógica de estas expresiones interpretadas descriptivamente (una lógica de proposiciones normativas), buscará reconstruir las calificaciones normativas de ciertas acciones de conformidad con un cierto conjunto de normas. De acuerdo con la conocida distinción de Hart, podría decirse que un sistema de lógica de normas refleja el punto de 
vista interno de quien dicta normas o quien las acepta y las usa como guía de conducta, mientras que un sistema de lógica de proposiciones normativas refleja el punto de vista externo de quien se sitúa como observador y califica normativamente ciertas acciones de conformidad con las normas existentes en un cierto sistema normativo. ${ }^{12}$

Una forma de explicar las diferencias entre una lógica de normas y una lógica de proposiciones normativas es a partir del diferente modo en el que la negación afecta a unas y otras. Aún cuando no se pueda decir que las normas sean verdaderas o falsas, la negación parece comportarse en el discurso prescriptivo de modo análogo a la negación ordinaria: la negación de una norma es también una norma; para cada norma sólo hay una norma que es su negación; una norma y su norma-negación ${ }^{13}$ son recíprocas (si “Op” es la negación de "P p", "P p" es la negación de "Op"). Además, una norma y su norma-negación son mutuamente excluyentes y conjuntamente exhaustivas, dado que " $\sim(\mathrm{Pp} \wedge \sim \mathrm{Pp})$ " y "Pp $\vee \sim \mathrm{Pp}$ " son admitidas como fórmulas válidas en la lógica de normas. ${ }^{14}$

La negación de las proposiciones normativas es más problemática. "En el sistema normativo $\alpha$ el acto $\mathrm{p}$ está prohibido" es un enunciado metalingüístico complejo que afirma que cierta norma pertenece a un sistema normativo, por lo que su negación puede ser entendida de dos modos diversos: "En el sistema $\alpha$ el acto $\mathrm{p}$ no está prohibido" puede interpretarse como expresivo de que el sistema $\alpha$ no contiene una norma que prohíbe la realización del acto $\mathrm{p}$, en cuyo caso la negación opera sobre la pertenencia de la norma al sistema -se niega la proposición normativa-. Pero también puede interpretárselo como significando que el sistema $\alpha$ contiene una norma que establece la no prohibición de $\mathrm{p}$ (esto es, una norma que permite $\mathrm{p}$ ), en cuyo caso la negación opera directamente sobre la norma. Para dar cuenta de esta ambigüedad es necesario diferenciar dos nociones de negación para las pro-

${ }^{12}$ Cf. H. L. A. Hart, El concepto de derecho, Buenos Aires, Abeledo Perrot, 1963, título original: The Concept of Law, Oxford, Oxford University Press, traducido por G. Carrió, páginas 110-111. En realidad, de acuerdo con Hart, no sólo es un enunciado externo aquel que describe el hecho de que una cierta regla existe, es decir, es aceptada por el grupo social, sino que también lo sería aquel que se limita a registrar las regularidades de conducta de quienes cumplen con las reglas y el que registra, además, la reacción hostil regular frente a las desviaciones del patrón usual de conducta (cf. H. L. A. Hart, obra citada, páginas 308-309 y E. Bulygin, "Norms, Normative Propositions and Legal Statements", en G. Floistad (ed.), Philosophy of Action. Chronicles of Philosophy, vol. III, The Hague, Martinus Nijhoff, 1982, páginas 107-125).

${ }^{13}$ La expresión "norma-negación" fue acuñada por G. H. von Wright en "Norms, Truth and Logic", en Practical Reason. Philosophical Papers, volume I, Oxford, Basil Blackwell, 1983, páginas 130-209. En un ejemplo, la norma-negación de "Op" es “ Op" (o "P p").

${ }^{14}$ Cf. C. Alchourrón, "Logic of Norms and Logic of Normative Propositions" y E. Bulygin, "Lógica Deóntica", ya citado. 
posiciones normativas: la negación externa (que se representará como “〜”) y la negación interna (que se representará como “ $\neg$ ”). Mientras la negación externa es una operación que lleva de la proposición normativa que afirma la pertenencia de una norma a un sistema a la proposición normativa que afirma la no pertenencia de tal norma al sistema, la negación interna es una operación que lleva de la proposición normativa que afirma la pertenencia de una norma a un sistema a la proposición normativa que afirma la pertenencia de su norma-negación al sistema. Por ejemplo:

Negación externa:

Negación interna:

$$
\begin{aligned}
& \sim \mathrm{PH}_{\alpha} \mathrm{p}=\operatorname{def} \text { "PHp" } \notin \alpha \\
& \neg \mathrm{PH}_{\alpha} \mathrm{p}=\operatorname{def} \text { “ } \sim \mathrm{PHp} " \in \alpha
\end{aligned}
$$

Esto es, la negación externa de la proposición que afirma que la conducta p está prohibida en el sistema normativo $\alpha$ equivale a negar la pertenencia de la norma que prohíbe $\mathrm{p}$ al sistema, mientras que la negación interna de tal proposición equivale a afirmar la pertenencia al sistema de una norma que establece la no prohibición de $\mathrm{p}$.

Ahora bien, adviértase que bajo una interpretación prescriptiva, decir que una conducta no está prohibida equivale a decir que está permitido realizarla. Bajo una interpretación descriptiva, en cambio, la expresión "permitido" se vuelve ambigua: una conducta puede ser calificada como permitida simplemente en razón de que nadie la ha prohibido, pero también puede calificársela como permitida en caso de que la autoridad normativa haya autorizado su realización. Estas dos nociones distintas, indiscernibles en el marco de una lógica de normas, pueden ser denominadas permisión negativa $\left(\mathrm{P}_{\alpha}^{-} \mathrm{p}\right)$ y permisión positiva $\left(\mathrm{P}_{\alpha}^{+} \mathrm{p}\right)$.

$$
\begin{array}{ll}
\text { Permisión negativa: } & \mathrm{P}_{\alpha}^{-} \mathrm{p}=\operatorname{def} \text { "PHp" } \notin \alpha \\
\text { Permisión positiva: } & \mathrm{P}_{\alpha}^{+} \mathrm{p}=\operatorname{def} \text { " } \sim \mathrm{PHp} " \in \alpha=\operatorname{def} \text { "Pp" } \in \alpha
\end{array}
$$

Como puede advertirse, la permisión negativa equivale a la negación externa de la prohibición de p, mientras que la permisión positiva equivale a la negación interna de la prohibición de p. La permisión negativa equivale a la no obligatoriedad de la abstención de una conducta, por lo cual se dirá que una conducta $\mathrm{p}$ está permitida en el sentido negativo en $\alpha$ sí, y sólo si, p no está prohibida en $\alpha$, es decir, si no existe en $\alpha$ una norma que prohíba $\mathrm{p}$. La permisión positiva depende, en cambio, de un acto llevado a cabo por la autoridad normativa, no de una mera omisión. En consecuencia, se dirá que una conducta está permitida en el sentido positivo en un sistema normativo $\alpha$ si, y sólo si, una norma que permite p pertenece a $\alpha$. 
La diferencia entre la permisión negativa y la permisión positiva quizás pueda entenderse con mayor claridad del siguiente modo: si una conducta p está permitida en sentido negativo en un sistema normativo y una autoridad decide prohibirla, no surgirá ningún conflicto. Como resultado de la prohibición, la conducta $\mathrm{p}$, antes no prohibida, pasará a estar prohibida en el sistema. Pero si la conducta $\mathrm{p}$ estaba permitida en sentido positivo y la autoridad decide prohibirla, surgirá en el sistema un conflicto normativo: $p$ estará permitida y prohibida a la vez.

Aunque la ambigüedad del término "permitido" fue advertida por von Wright, el profesor finlandés consideró por cierto tiempo que la permisión positiva tenía carácter normativo, mientras que la negativa no. ${ }^{15}$ En otras palabras, von Wright distinguía sólo dos conceptos de permisión, dado que identificaba la permisión prescriptiva con la permisión positiva. Y como suponía que la permisión positiva implicaba a la negativa, denominaba a la primera "permisión fuerte" y a la segunda "permisión débil". En realidad, resulta necesario diferenciar tres conceptos de permisión, uno prescriptivo y dos descriptivos: el permiso positivo y el negativo. Es más: para una lógica de proposiciones normativas se puede tomar como base al operador obligatorio (o al operador prohibido) de la lógica de normas y definir a partir de él dos conceptos descriptivos de permisión y dos de facultamiento, pero también podría tomarse como base al operador permitido y definir a partir de él dos conceptos de prohibición y dos de obligación:

Base O:

(1) $\mathrm{O}_{\alpha} \mathrm{p}=\operatorname{def}$ "Op" $\in \alpha$

(2) $\mathrm{PH}_{\alpha} \mathrm{p}=\operatorname{def}$ "O $\sim \mathrm{p}$ " $\in \alpha$

(3) $\mathrm{P}_{\alpha}^{+} \mathrm{p}=\operatorname{def} " \sim 0 \sim \mathrm{p}$ " $\in \alpha$

(3') $\mathrm{P}_{\alpha}^{-} \mathrm{p}=\operatorname{def}$ "O $\mathrm{p}$ " $\notin \alpha$

(4) $\mathrm{F}^{+} \mathrm{p}=\operatorname{def}$ “ $\sim \mathrm{Op} " \in \alpha \wedge$ “ $\sim \sim \mathrm{p}$ " $\in \alpha$

(4') $\mathrm{F}^{-} \mathrm{p}=\operatorname{def}$ “Op" $\notin \alpha \wedge$ “O $\sim \mathrm{p}$ ” $\notin \alpha$ $\underline{\text { Base P: }}$

(1") $\mathrm{O}_{\alpha}^{+} \mathrm{p}=\operatorname{def} " \sim \mathrm{P} \sim \mathrm{p}$ " $\in \alpha$

(1') $\mathrm{O}_{\alpha}^{-} \mathrm{p}=\operatorname{def}$ "P $\sim \mathrm{p}$ " $\notin \alpha$

(2") $\mathrm{PH}_{\alpha}^{+} \mathrm{p}=\mathrm{def}$ “ $\sim \mathrm{Pp} " \in \alpha$

(2') $\mathrm{PH}_{\alpha}^{-} \mathrm{p}=\operatorname{def}$ "Pp" $\notin \alpha$

(3") $\mathrm{P}_{\alpha} \mathrm{p}=\operatorname{def}$ "Pp" $\in \alpha$

(4") $\mathrm{F}_{\alpha} \mathrm{p}=\operatorname{def}$ "Pp" $\in \alpha \wedge$ "P p" $\in \alpha$

Las fórmulas identificadas con números seguidos de comillas dobles equivalen a las numeradas sin comillas de la otra presentación, mientras que las numeradas con una comilla no tienen equivalente en la versión alternativa. De esto resulta que, desde un punto de vista formal, a partir de la ambigüedad de la negación de las proposiciones normativas, pueden dis102

${ }^{15}$ Cf. G. H. von Wright, Norma y Acción. Una investigación lógica, ya citado, página 
tinguirse los siguientes cuatro pares de conceptos, con sus correspondientes equivalencias:
(1) $\mathrm{O}_{\alpha}^{+} \mathrm{p}=\sim \mathrm{P}_{\alpha}^{-} \sim \mathrm{p}=\neg \mathrm{P}^{+} \sim \mathrm{p}$
(1') $\mathrm{O}_{\alpha}^{-} \mathrm{p}=\sim \mathrm{P}_{\alpha}^{+} \sim \mathrm{p}=\neg \mathrm{P}^{-}{ }_{\alpha}^{-} \sim \mathrm{p}$
(2) $\mathrm{PH}_{\alpha}^{+} \mathrm{p}=\sim \mathrm{P}_{\alpha}^{-} \mathrm{p}=\neg \mathrm{P}^{+}{ }_{\alpha} \mathrm{p}$
(2') $\mathrm{PH}_{\alpha}^{-} \mathrm{p}=\sim \mathrm{P}_{\alpha}^{+} \mathrm{p}=\neg \mathrm{P}^{-}{ }_{\alpha} \mathrm{p}$
(3) $\mathrm{P}_{\alpha}^{+} \mathrm{p}=\sim \mathrm{PH}^{-}{ }_{\alpha} \mathrm{p}=\neg \mathrm{PH}^{+} \mathrm{p}$
(3') $\mathrm{P}^{-}{ }_{\alpha} \mathrm{p}=\sim \mathrm{PH}^{+}{ }_{\alpha} \mathrm{p}=\neg \mathrm{PH}^{-}{ }_{\alpha} \mathrm{p}$
(4) $\mathrm{F}_{\alpha}^{+} \mathrm{p}=\sim \mathrm{O}_{\alpha}^{-} \mathrm{p} \wedge \sim \mathrm{O}_{\alpha}^{-} \sim \mathrm{p}=\neg \mathrm{O}_{\alpha}^{+} \mathrm{p} \wedge \neg \mathrm{O}_{\alpha}^{+} \sim \mathrm{p}$
(4') $\mathrm{F}^{-} \mathrm{p}=\sim \mathrm{O}^{+}{ }_{\alpha} \mathrm{p} \wedge \sim \mathrm{O}^{+} \sim \mathrm{p}=\neg \mathrm{O}_{\alpha}^{-} \mathrm{p} \wedge$ $\neg \mathrm{O}_{\alpha}^{-} \sim \mathrm{p}$

Sin embargo, los conceptos de prohibición negativa $\left(\mathrm{PH}_{\alpha}^{-}\right)$y obligación negativa $\left(\mathrm{O}_{\alpha}^{-}\right)$no parecen tener correlato en el lenguaje corriente, razón por la cual se utiliza en general una presentación sobre la base del operador obligatorio y se habla exclusivamente de dos conceptos descriptivos de permisión. ${ }^{16}$

El primer desarrollo de una lógica de proposiciones normativas se debe a Carlos Alchourrón. ${ }^{17} \mathrm{Su}$ propuesta podría presentarse axiomáticamente del siguiente modo:

\section{Sistema LPN:}

$$
\begin{array}{ll}
\left(\mathrm{A}_{1}\right) & -\mathrm{O}_{\alpha}(\mathrm{p} \wedge \mathrm{q}) \leftrightarrow\left(\mathrm{O}_{\alpha} \mathrm{p} \wedge \mathrm{O}_{\alpha} \mathrm{q}\right) \\
\left(\mathrm{A}_{2}\right) & -\mathrm{O}_{\alpha} \mathrm{p} \rightarrow \mathrm{P}_{\alpha}^{+} \mathrm{p} \\
\left(\mathrm{A}_{3}\right) & -\mathrm{P}_{\alpha}^{+}(\mathrm{p} \wedge \mathrm{q}) \rightarrow \mathrm{P}_{\alpha}^{+} \mathrm{p} \\
& \\
\left(\mathrm{RI}_{1}\right) & \text { De } \mid-(\mathrm{p} \leftrightarrow \mathrm{q}), \text { se sigue } \mid-\left(\mathrm{O}_{\alpha} \mathrm{p} \leftrightarrow \mathrm{O}_{\alpha} \mathrm{q}\right) \\
\left(\mathrm{RI}_{2}\right) & \text { De } \mid-(\mathrm{p} \leftrightarrow \mathrm{q}), \text { se sigue } \mid-\left(\mathrm{P}_{\alpha}^{+} \mathrm{p} \leftrightarrow \mathrm{P}_{\alpha}^{+} \mathrm{q}\right)^{18}
\end{array}
$$

De la comparación entre este sistema y el sistema de lógica de normas (LN) surge, en primer lugar, que las proposiciones normativas son relativas a un determinado sistema normativo, lo cual se refleja en la aparición de los suscriptos $\left({ }_{\alpha}\right)$ en las fórmulas correspondientes. Por contraste, las expresiones de la lógica de normas no están referidas a un sistema normativo, sino

16 “(...) podríamos definir también la noción de prohibición débil, como la ausencia (en $\alpha$ ) de una norma que permita p. Pero esta noción es muy poco usada: sólo en circunstancias muy especiales una conducta se calificaría como prohibida por el mero hecho de que no estuviera expresamente permitida. Menos usual todavía es la noción de obligación débil” (C. Alchourón y E. Bulygin, Introducción a la metodología de las ciencias jurídicas y sociales, Buenos Aires, Astrea, 1975, título original: Normative Systems, Wien-New York, Springer Verlag, 1971, traducido por los autores, página 177, nota).

${ }^{17}$ Cf. C. Alchourrón, "Logic of Norms and Logic of Normative Propositions", ya citado.

${ }^{18}$ Cf. C. Alchourrón, "Philosophical Foundations of Deontic Logic and the Logic of Defeasible Conditionals", ya citado. 
que se trata en este sentido de conceptos absolutos. En segundo lugar, en el sistema de lógica de proposiciones normativas no existe nada parecido al axioma $\mathrm{A}_{0}$ del sistema LN. Por otro lado, en el sistema LPN hay un axioma y una regla de inferencia $\left(\mathrm{A}_{3} \mathrm{y} \mathrm{RI}_{2}\right)$ que no se encuentran en LN. No obstante, esta última es una diferencia menor: si se pudiera agregar al sistema de lógica de proposiciones normativas un análogo del axioma $\mathrm{A}_{0}$ de $\mathrm{LN}, \mathrm{A}_{3}$ y $\mathrm{RI}_{2}$ resultarían superfluos por cuanto serían deducibles de los restantes axiomas. El punto en discordia es, pues, el axioma $\mathrm{A}_{0}$.

¿Por qué razón $A_{0}$ no puede tomarse como axioma en un sistema de lógica para proposiciones normativas? Porque, como se ha visto, hay dos formas distintas en las que una proposición normativa puede ser negada, las cuales originan dos conceptos descriptivos para cada uno de los operadores deónticos. Y aunque la negación externa satisface todos los requisitos que se espera que cumpla una negación -los mismos cinco principios que se enunciaron en formulación analógica como satisfechos por la negación de las normas-, ello no ocurre con la negación interna. Una proposición normativa y su negación interna pueden ser ambas verdaderas -en cuyo caso el sistema será inconsistente- tanto como ambas falsas -en cuyo caso el sistema será incompleto-. ${ }^{19}$ De ahí que en la lógica de proposiciones normativas no sea válida la equivalencia expresada por el axioma $\mathrm{A}_{0}$ de la lógica de normas.

Pese a las diferencias apuntadas, puede demostrarse que bajo ciertos presupuestos los sistemas LN y LPN serían equivalentes. Si un sistema normativo satisface las dos condiciones siguientes respecto de cualquier conducta $\mathrm{p}$, puede probarse que sería válido para LPN un análogo del axioma $\mathrm{A}_{0}$ de $\mathrm{LN}$ o, lo que es lo mismo, que la permisión positiva $\mathrm{P}^{+}$equivaldría a la permisión negativa $\mathrm{P}^{-}$:
12. $\sim\left(\mathrm{O}_{\alpha} \sim \mathrm{p} \wedge \mathrm{P}_{\alpha}^{+} \mathrm{p}\right)$
(Cons $\left.{ }_{\alpha} \mathrm{p}\right)$
13. $\mathrm{O}_{\alpha} \sim \mathrm{p} \vee \mathrm{P}^{+}{ }_{\alpha} \mathrm{p}$
$\left(\mathrm{Comp}_{\alpha} \mathrm{p}\right)$

Ello en virtud de que

14. $\sim\left(\mathrm{O}_{\alpha} \sim \mathrm{p} \wedge \mathrm{P}_{\alpha}^{+} \mathrm{p}\right) \wedge \mathrm{O}_{\alpha} \sim \mathrm{p} \vee \mathrm{P}_{\alpha}^{+} \mathrm{p}$

equivale a

15. $\mathrm{P}_{\alpha}^{+} \mathrm{p} \leftrightarrow \sim \mathrm{O}_{\alpha} \sim \mathrm{p}$

${ }^{19}$ Cf. E. Bulygin, "Lógica Deóntica”, ya citado. 
Demostración: ${ }^{20}$

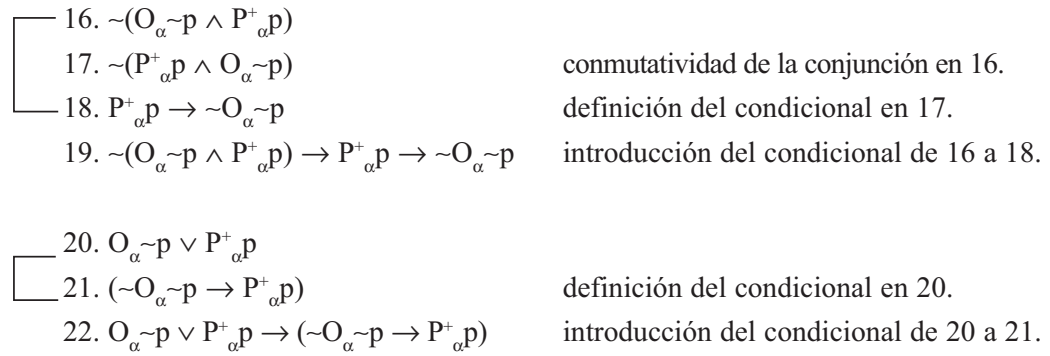

De esto se sigue que:

23. $\left(\left(\right.\right.$ Cons $\left.\left._{\alpha} \mathrm{p}\right) \wedge\left(\mathrm{Comp}_{\alpha} \mathrm{p}\right)\right) \leftrightarrow\left(\mathrm{P}_{\alpha}^{+} \mathrm{p} \leftrightarrow \sim \mathrm{O}_{\alpha} \sim \mathrm{p}\right)$

y dado que

24. $\sim \mathrm{O}_{\alpha} \sim \mathrm{p} \leftrightarrow \mathrm{P}_{\alpha}^{-} \mathrm{p}$

entonces:

25. $\left(\left(\right.\right.$ Cons $\left.\left._{\alpha} \mathrm{p}\right) \wedge\left(\mathrm{Comp}_{\alpha} \mathrm{p}\right)\right) \leftrightarrow\left(\mathrm{P}^{+}{ }_{\alpha} \mathrm{p} \leftrightarrow \mathrm{P}^{-}{ }_{\alpha} \mathrm{p}\right)$

En otras palabras, bajo el supuesto de completitud y consistencia, el sistema LPN se comporta de modo semejante al sistema LN. Igualmente, podría decirse que un sistema de lógica de normas equivale a un sistema de lógica de proposiciones normativas para un sistema completo y consistente. Una lógica de normas pretendería reconstruir la racionalidad en el dictado de normas, y un legislador racional no debería contradecirse ni dejar conductas sin determinar normativamente. Por el contrario, desde una lógica de proposiciones normativas no puede suponerse la completitud ni la consistencia del sistema al que se hace referencia, puesto que de hecho un sistema normativo puede contener lagunas y contradicciones.

Finalmente, puede demostrarse que:

Proposición 1: Si un sistema normativo es inconsistente respecto de la acción $\mathrm{p}$, entonces es completo respecto de la acción $\mathrm{p}:\left(\sim\left(\mathrm{Cons}_{\alpha} \mathrm{p}\right) \rightarrow\right.$ (Comp $\left.\left.{ }_{\alpha} \mathrm{p}\right)\right)$.

26. $\mathrm{O}_{\alpha} \sim \mathrm{p} \wedge \mathrm{P}_{\alpha}^{+} \mathrm{p}$

27. $\mathrm{O}_{\alpha} \sim \mathrm{p}$

eliminación de la conjunción en 26.

28. $\mathrm{O}_{\alpha} \sim \mathrm{p} \vee \mathrm{P}^{+}{ }_{\alpha} \mathrm{p}$ introducción de la disyunción en 27.

29. $\left(\mathrm{O}_{\alpha} \sim \mathrm{p} \wedge \mathrm{P}_{\alpha}^{+} \mathrm{p}\right) \rightarrow\left(\mathrm{O}_{\alpha} \sim \mathrm{p} \vee \mathrm{P}_{\alpha}^{+} \mathrm{p}\right) \quad$ introducción del condicional de 26 a 28.

\footnotetext{
${ }^{20}$ Aquí, como en las demostraciones de la página siguiente, doy por conocidas las reglas básicas del cálculo de deducción natural de Gentzen (cf. G. Gentzen, "Untersuchungen über das Logische Schliessen”, en Mathematische Zeitschrift 39, 1934, páginas 176-210).
} 
Proposición 2: Si un sistema normativo es incompleto respecto de la acción $\mathrm{p}$, entonces es consistente respecto de la acción $\mathrm{p}:\left(\sim\left(\operatorname{Comp}_{\alpha} \mathrm{p}\right) \rightarrow\right.$ $($ Cons $\mathrm{p}$ ) ).

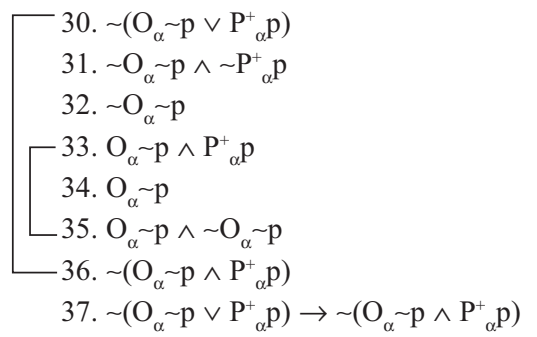

\author{
De Morgan en 30. $(\sim(p \vee q) \leftrightarrow(\sim p \wedge \sim q))$. \\ eliminación de la conjunción en 31 . \\ eliminación de la conjunción en 33 . \\ introducción de la conjunción en 32 y 34. \\ introducción de la negación de 33 a 35 . \\ introducción del condicional de 30 a 36.
}

Estas dos demostraciones pueden generalizarse debido a la aplicación del principio ex falso sequitur quodlibet $((\mathrm{p} \wedge \sim \mathrm{p}) \mid-\mathrm{q})$, de lo que se sigue:

Proposición 3: Si un sistema normativo es inconsistente respecto de una cierta acción, entonces es completo respecto de cualquier acción ( $\exists \mathrm{x}$ $\sim\left(\right.$ Cons $\left.\left._{\alpha} \mathrm{x}\right) \rightarrow \forall \mathrm{x}\left(\operatorname{Comp}_{\alpha} \mathrm{x}\right)\right)$.

Proposición 4: Si un sistema normativo es incompleto respecto de una cierta acción, entonces es consistente respecto de cualquier acción ( $\exists \mathrm{x}$ $\sim\left(\operatorname{Comp}_{\alpha} \mathrm{x}\right) \rightarrow \forall \mathrm{x}\left(\right.$ Cons $\left.\left._{\alpha} \mathrm{x}\right)\right)$.

\section{Dos visiones de la lógica de proposiciones normativas}

Pese a que, como se dijo, ni a la distinción entre normas y proposiciones normativas, ni mucho menos a la diferencia entre una genuina lógica de normas y una lógica de proposiciones normativas, se les ha reconocido la importancia que a mi criterio ellas merecen, puede decirse que hasta no hace mucho tiempo el sistema de lógica de proposiciones normativas propuesto por Alchourrón, y desarrollado posteriormente por él mismo en colaboración con Bulygin en diversos estudios, reinaba como clásico sin tener que disputar su lugar con ninguna propuesta alternativa.

Sin embargo, en un breve y reciente trabajo, von Wright presentó un camino diferente para reconstruir una lógica de proposiciones normativas. ${ }^{21}$ De acuerdo con este sistema, la forma esquemática para enunciados deónticos consistiría en:

a) una variable de enunciado o compuesto molecular de variables de enunciado a las cuales se ha antepuesto un operador deóntico O o P, o

b) un compuesto molecular de enunciados como los definidos en a).

${ }^{21}$ Cf. G. H. von Wright, "On norms and Norm-Propositions. A Sketch”, en W. Krawietz et al., The Reasonable as Rational? On Legal Argumentation and Justification. Festschrift for Aulis Aarnio, Berlin, Duncker \& Humblot, 2000, páginas 173-178. 
Bajo una lectura descriptiva, los enunciados deónticos expresarían proposiciones normativas, y para ellas valdrían las leyes de la lógica de enunciados (proposicional) clásica, así como también un principio según el cual compuestos lógicamente equivalentes de variables de enunciado son sustituibles salva veritate. No se presupondría que valga ninguna verdad lógica especial para las proposiciones normativas. En particular, una serie de fórmulas que en su lectura prescriptiva son consideradas válidas (esto es, en el marco de una lógica de normas), no resultarían aquí lógicamente verdaderas. Entre ellas:

$$
\begin{aligned}
& \text { 38. } \sim \mathrm{O} \sim \mathrm{p} \rightarrow \mathrm{Pp} \\
& \text { 39. } \mathrm{Pp} \rightarrow \sim \mathrm{O} \sim \mathrm{p} \\
& \text { 40. } \mathrm{Pp} \vee \mathrm{P} \sim \mathrm{p} \\
& \text { 41. } \mathrm{O}(\mathrm{p} \wedge \mathrm{q}) \rightarrow \sim \mathrm{P} \sim \mathrm{p} \\
& \text { 42. } \mathrm{P}(\mathrm{p} \wedge \mathrm{q}) \rightarrow \sim \mathrm{O} \sim \mathrm{p}
\end{aligned}
$$

Es fácil advertir, tal como el propio von Wright lo señala, que (38) equivale al principio de completitud y (39) al principio de consistencia. Como se examinó en el punto anterior, ninguno de estos principios, sin calificaciones, es admitido por Alchourrón en su sistema de lógica de proposiciones normativas. Se trata en ambos casos de características contingentes de los sistemas normativos.

Sin embargo, como también puntualiza von Wright, (39) equivale a "Op $\rightarrow \sim \mathrm{P} \sim \mathrm{p}$ " y (40) a " $\sim \mathrm{P} \sim \mathrm{p} \rightarrow \mathrm{Pp}$ ". De manera que, conjuntamente, implican por transitividad "Op $\rightarrow \mathrm{Pp}$ ", cuya satisfacción por un sistema normativo sería entonces también contingente. Aquí "Pp" debería interpretarse, en la lectura descriptiva propuesta, como " $\mathrm{P}_{\alpha}^{+} \mathrm{p}$ ", esto es, como una expresión referida a la permisión positiva. Ahora bien, esto choca con la asunción, en el sistema de Alchourrón, del axioma $\mathrm{A}_{2}$. Por su parte, (41) en conjunción con (38) y (39) implica " $\mathrm{O}(\mathrm{p} \wedge \mathrm{q}) \rightarrow(\mathrm{Op} \wedge \mathrm{Oq})$ ", que constituye el axioma $\mathrm{A}_{1}$ del sistema de Alchourrón. Y (42) en conjunción con (38) implica " $P(p \wedge q) \rightarrow$ Pp", esto es, el axioma $\mathrm{A}_{3}$ del sistema de Alchourrón. En consecuencia, si tal como lo sostiene von Wright ninguna de las fórmulas listadas de (38) a (42) fuesen lógicamente verdaderas, ¡no quedaría en pie ninguno de los axiomas que Alchourrón asume para una lógica de proposiciones normativas!

Esto, que a primera vista parece tan sorprendente, no debería en realidad llamar nuestra atención, siendo que von Wright advierte, como se adelantó, que en su sistema no vale ninguna verdad lógica especial para las proposiciones normativas. Lo notable, en todo caso, lo que requiere de alguna justificación, es la profunda diferencia de enfoques que parece signar a uno y otro sistema. 
Un primer paso para intentar explicar esa diferencia consiste en tomar en cuenta la distinción entre lo que Alchourrón y Bulygin han denominado concepción hilética y concepción expresiva de las normas. ${ }^{22}$ De acuerdo con la concepción hilética, las normas son entendidas como el significado de ciertos enunciados - las formulaciones normativas-, por lo que se trataría de entidades semejantes a las proposiciones. Las formulaciones normativas constituirían expresiones lingüísticas de normas y las normas serían los significados de tales enunciados prescriptivos, así como las proposiciones son - para ciertos autores- el significado de los enunciados descriptivos. La diferencia radicaría en que los enunciados descriptivos indican que algo es de una cierta manera, mientras que los enunciados prescriptivos indican que algo debe o no debe o puede ser hecho. De acuerdo con la concepción expresiva, las normas son el resultado del uso prescriptivo del lenguaje. Un mismo enunciado podría ser usado en diferentes ocasiones para hacer cosas distintas: aseverar, ordenar, interrogar, etc. El resultado de esas acciones sería una aserción, una orden o una pregunta, etc., por lo que tan sólo en el nivel pragmático del uso del lenguaje se localizaría la diferencia entre aserciones, órdenes y preguntas, etc., no en el nivel semántico.

Entre los partidarios de la concepción hilética hay quienes consideran que las normas son susceptibles de verdad o falsedad y, consiguientemente, aceptan la posibilidad de una lógica de normas junto a una lógica de proposiciones normativas. La lógica de normas se ocuparía de las relaciones entre proposiciones prescriptivas, mientras que la lógica de proposiciones normativas se ocuparía de las relaciones lógicas entre las proposiciones descriptivas acerca de normas. Otros partidarios de esta concepción, en cambio, no aceptan que se pueda hablar de verdad o falsedad de las normas, no obstante lo cual creen en la posibilidad de una lógica de normas, pero para ello es necesario concebir a la lógica como no circunscripta exclusivamente a enunciados verdaderos o falsos. Podría decirse entonces que la creencia en la existencia de relaciones lógicas entre normas no compromete con la aceptación de que las normas sean verdaderas o falsas. Para los partidarios de la concepción expresiva en principio sólo es posible una lógica de proposiciones normativas, es decir, de enunciados que describen normas. Desde esta perspectiva, no habría lógica de normas sin referencia al sistema normativo del cual tales normas forman parte. ${ }^{23}$

${ }^{22}$ Cf. C. Alchourrón y E. Bulygin, "The Expressive Conception of Norms", en R. Hilpinen (ed.), New Studies in Deontic Logic, Dordrecht-Boston-London, Reidel, 1981, páginas 95-124.

${ }^{23}$ Cf. D. Makinson, “On a Fundamental Problem of Deontic Logic”, en P. McNamara y H. Prakken (eds.), Norms, Logics and Information Systems. New Studies on Deontic Logic and Computer Science, Amsterdam, IOS Press, 1998. 
Según Alchourrón y Bulygin, adoptar la concepción expresiva de las normas no condenaría al discurso normativo a un completo irracionalismo, puesto que la racionalidad podría preservarse en el plano de las proposiciones normativas. Si bien puede admitirse esta idea, es importante destacar que la lógica de proposiciones normativas que desarrollan Alchourrón y Bulygin respecto de la concepción expresiva de las normas es sustancialmente idéntica a la que atribuyen a la concepción alternativa, esto es, a la concepción hilética. Ambas responden al modelo LPN reseñado en el punto anterior, y en ambas se presupone una noción de sistema normativo clausurado bajo la noción de consecuencia. Así, en "La concepción expresiva de las normas", los autores argentinos sostienen:

"De esta manera, la existencia de una norma (= pertenencia del contenido normativo al conjunto ordenado) depende de ciertos hechos empíricos (actos de promulgación en el caso de las prescripciones; ciertas acciones reveladoras de disposiciones en el caso de las normas consuetudinarias). Por lo tanto, como no hay relaciones lógicas entre hechos, no hay cabida para una lógica de normas. Pero esto no precluye la posibilidad de una lógica de proposiciones normativas. En efecto, tal como ya lo hemos señalado, la proposición de que $\mathrm{p}$ es obligatorio en A es verdadera si p ha sido ordenado por Rex y pertenece, por lo tanto, al conjunto ordenado A. Pero esto es una condición suficiente, pero no necesaria para la verdad de la proposición " $p$ es obligatorio en A". Puede ocurrir que p no haya sido nunca ordenado por Rex y, sin embargo, $p$ sea obligatorio en A. Supongamos, por ejemplo, que Rex no haya ordenado p, pero sí ha ordenado p\&q. Esta es una proposición diferente de $\mathrm{p} y$, conforme a nuestro criterio, $\mathrm{p}$ no pertenecería a A. Pero como $p$ es una consecuencia lógica de $p \& q$ (pues $p \& q$ implica lógicamente $\mathrm{p}$ ), también es verdad que $\mathrm{p}$ es obligatorio en A. La obligatoriedad de $\mathrm{p}$ es una consecuencia lógica de la obligatoriedad de $\mathrm{p} \& \mathrm{q}$, porque $\mathrm{p}$ es una consecuencia lógica de $\mathrm{p} \& \mathrm{q}$. Podemos definir ahora el concepto de sistema normativo como el conjunto de todas las proposiciones que son consecuencias lógicas de las proposiciones ordenadas expresamente. (Aunque estemos usando la expresión tradicional "sistema normativo", hay que tener presente que en la concepción expresiva un sistema normativo no es un conjunto de normas, sino un conjunto de contenidos normativos, esto es, de proposiciones). Esto hace posible distinguir entre el conjunto A (formado por todas las proposiciones expresamente ordenadas) como la base axiomática del sistema y el sistema normativo $\mathrm{Cn}(\mathrm{A})$, que es el conjunto de las consecuencias de A. Estamos en condiciones de poder corregir nuestro criterio de verdad para las proposiciones normativas: " $p$ es obligatorio en A" es verdadera si, y sólo si, p pertenece al sistema $\mathrm{Cn}(\mathrm{A})$, es decir, si, y sólo si, p pertenece a las consecuencias de A". ${ }^{24}$

Ahora bien, si la reconstrucción de una lógica de proposiciones normativas ha de servir tanto a un partidario de la concepción hilética como a un partidario de la concepción expresiva, la asunción de una noción de sistema normativo clausurado bajo la noción de consecuencia parece inadmisible,

\footnotetext{
${ }^{24}$ C. Alchourrón y E. Bulygin, "The Expressive Conception of Norms”, ya citado.
} 
puesto que el expresivista no admitiría relaciones lógicas entre normas. El modo en el que Alchourrón y Bulygin intentan salvar esta dificultad al presentar su visión de la lógica de proposiciones normativas en el marco de la concepción expresiva consiste en no reconocer directamente relaciones lógicas entre normas, pero interpretar al "sistema normativo" como un conjunto de contenidos normativos, conjunto que sí se hallaría clausurado bajo la noción de consecuencia. No obstante, con esto las dos versiones de la lógica de proposiciones normativas confluyen en el sistema LPN.

¿Qué pasaría, en cambio, si se abandona ese presupuesto? En LPN, como vimos, hay verdades lógicas específicas para las proposiciones normativas: que obligatorio $\mathrm{p}$ en el sistema normativo $\alpha$ implica permitido en sentido positivo $\mathrm{p}$ en $\alpha$; o que la obligatoriedad de la conjunción de $\mathrm{p}$ y q en $\alpha$ equivale a sostener que es obligatorio $\mathrm{p}$ y es obligatorio q en $\alpha$; o que la permisión positiva de la conjunción de $\mathrm{p}$ y q en $\alpha$ implica la permisión de cualquiera de los conjuntos en $\alpha$. Ahora bien, si se piensa en una lógica de proposiciones normativas relativa a un sistema que no comprende todas sus consecuencias lógicas, no sólo debería admitirse que un sistema semejante puede ser inconsistente o incompleto (porque prohibido $\mathrm{p}$ en $\alpha$ y permitido en sentido positivo $\mathrm{p}$ en $\alpha$ pueden ser ambas verdaderas tanto como ambas falsas), al igual que en LPN, sino además que podría ser verdad que es obligatorio $\mathrm{p}$ en $\alpha \mathrm{y}$, no obstante, no estar permitido en sentido positivo $\mathrm{p}$ en $\alpha$; o que sea obligatorio p y q en $\alpha$ y no sea obligatorio $\mathrm{p}$ en $\alpha$; o que sea verdad que está permitido $\mathrm{p}$ y q en $\alpha$ y no esté permitido $\mathrm{p}$ en $\alpha$. Que se satisfagan o no principios tales como que obligatorio implica permitido, o la distributividad de la obligación o la permisión por la conjunción, sería una cuestión tan contingente como la satisfacción de la completitud y la consistencia.

Esto es exactamente lo que ocurre en el sistema que esboza von Wright. A diferencia de Alchourrón y Bulygin, von Wright toma en cuenta proposiciones normativas relativas a un sistema no clausurado bajo la noción de consecuencia, sino sólo compuesto por las normas de base, esto es, las expresamente dictadas. El sistema de von Wright se limita a describir actos normativos, y del hecho de que se haya dictado en $\alpha$ la norma "Op", por ejemplo, no se sigue que se haya dictado en $\alpha$ la norma "Pp". En la lógica de normas, "Op" puede definirse como "Pp $\wedge \sim \mathrm{P} \sim \mathrm{p}$ ", y ciertamente "Op" implica "Pp". Pero el operador descriptivo "O $\mathrm{p}^{\mathrm{p}}$ ( (es obligatorio en el sistema $\alpha$ la acción $p$ ) equivale a "Op" $\in \alpha$, donde la norma "Op" está siendo mencionada, no usada. Por eso, de "Op" $\in \alpha$ no se sigue que "Pp" $\in \alpha$, salvo que se presuponga que $\alpha$ no está sólo conformado por las normas expresamente dictadas, sino que comprende todas sus consecuencias lógicas $(\operatorname{Cn}(\alpha)=\alpha)$. En otras palabras, del hecho de que una acción sea calificada como obligatoria según las normas del sistema $\alpha$ se sigue que dicha acción 
está permitida en sentido positivo en $\alpha$ sólo bajo el presupuesto de clausura bajo la noción de consecuencia deductiva. Lo mismo ocurre con la distributividad de la obligación o la permisión por la conjunción.

Estas dos alternativas de reconstrucción de la lógica de proposiciones normativas se corresponden entonces con dos diferentes concepciones de los sistemas normativos: una según la cual se los reconstruye como exclusivamente compuestos por las normas de base, y otra según la cual se los reconstruye como la totalidad de las consecuencias que se siguen de ciertas normas de base. Puede resultar instructivo considerar en este punto cómo se encara un problema semejante en el terreno de los estudios lógicos sobre dinámica de creencias. En dicho campo es usual el empleo alternativo de dos estrategias diferentes para enfocar el análisis, a las que se denomina, respectivamente, bases de creencias y teorías. De acuerdo con esta distinción, A es una base de creencias para una teoría $\mathrm{K}$ si y sólo si $\mathrm{K}=\mathrm{Cn}(\mathrm{A})$. En otros términos, una teoría es un conjunto de creencias que comprende todas sus consecuencias lógicas, mientras que una base de creencias es un conjunto no clausurado bajo la noción de consecuencia. ${ }^{25}$ Partir de bases de creencias y no de teorías ofrecería ciertas ventajas, puesto que las bases son finitas, lo que permite su tratamiento computacional, y poseen además mayor poder expresivo que las teorías. No obstante, el enfoque de las bases de creencias tiene la desventaja de que es muy difícil diferenciar entre creencias básicas y derivadas. Este problema, sin embargo, no se presenta respecto de ciertos sistemas normativos, tales como los sistemas jurídicos, a cuyo respecto existen criterios convencionalmente aceptados para diferenciar entre normas de base y normas derivadas a partir de las primeras.

El sistema de von Wright parece el más adecuado para dar cuenta de proposiciones normativas respecto de un sistema de normas en el que éstas se conciben como actos de prescribir y, por consiguiente, que no admite relaciones lógicas entre las propias normas. E incluso desde este punto de vista también es posible preservar la idea que subyace a los desarrollos de lógicas de normas, interpretándolos como sistemas de lógica de proposiciones normativas relativas a un conjunto de normas dictado por un legislador racional. ${ }^{26}$

Marzo de 2004

${ }^{25}$ Cf. P. Gärdenfors, “The Dynamics of Normative Systems”, en A. Martino (ed.), Expert Systems in Law, Elsevier Science Publishers B.V., 1992, páginas 195-200 y S. O. Hansson, A Textbook of Belief Dynamics. Theory Change and Database Updating, Dordrecht-Boston-London, Kluwer Academic Publishers, 1999.

${ }^{26}$ Cf. G. H. von Wright, "Norms, Truth and Logic", ya citado.

\section{DOXA 26 (2003)}

